\title{
Evidence for Decreased Splanchnic Glucose Uptake after Oral Glucose Administration in Non-Insulin-dependent Diabetes Mellitus
}

\author{
Bernhard Ludvik, ${ }^{\star}$ John J. Nolan,, Anne Roberts, ${ }^{\ddagger}$ Joseph Baloga, ${ }^{*}$ Mary Joyce, ${ }^{\star}$ Jo M. Bell, ${ }^{\star}$ and Jerrold M. Olefsky* \\ $*$ Department of Medicine and ${ }^{\ddagger}$ Department of Radiology, University of California San Diego and Veterans Administration Hospital, \\ San Diego, California 92161
}

\begin{abstract}
The role of splanchnic glucose uptake (SGU) after oral glucose administration as a potential factor contributing to postprandial hyperglycemia in non-insulin-dependent diabetes mellitus (NIDDM) has not been established conclusively. Therefore, we investigated SGU in six patients with NIDDM and six weight-matched control subjects by means of the hepatic vein catheterization (HVC) technique. In a second part, we examined the applicability of the recently developed OG-CLAMP technique in NIDDM by comparing SGU and first-pass SGU during HVC with SGU during the OGCLAMP experiment. The OG-CLAMP method combines a euglycemic, hyperinsulinemic clamp and an oral glucose tolerance test (75 g) during steady state glucose infusion (GINF). During HVC, SGU equals the splanchnic fractional extraction times the total (oral and arterial) glucose load presented to the liver. For OG-CLAMP, SGU was calculated as first-pass SGU by subtracting the integrated decrease in GINF over $180 \mathrm{~min}$ from $75 \mathrm{~g}$. Cumulative splanchnic glucose output after oral glucose correlated significantly between both methods and was increased significantly in NIDDM patients $(73.1 \pm 5.1 \mathrm{~g}$ for $\mathrm{HVC}, \mathbf{7 6 . 5} \pm 5.5$ for OG-CLAMP) compared with nondiabetic patients $(46.7 \pm 4.4 \mathrm{~g}$ for $\mathrm{HVC}$, 57.5 \pm 1.9 for OG-CLAMP). Thus, in NIDDM patients, SGU (7.4 \pm 2.1 vs. $37.8 \pm 5.9 \%$ in nondiabetic patients, $P<0.001)$ and first-pass SGU $(4.7 \pm 1.7$ vs. $26.5 \pm 5.1 \%$ in nondiabetic patients, $P<0.01$ ) were decreased significantly during $\mathrm{HVC}$, as was SGU during OG-CLAMP (3.9 \pm 1.7 vs. $23.4 \pm 2.5 \%$ in nondiabetic patients, $P<0.0001)$. SGU measured during OG-CLAMP correlated significantly with SGU $(r=0.87, P<$ 0.05 for NIDDM patients; $r=0.94, P<0.01$ for nondiabetic patients) and first-pass SGU $(r=0.87, P<0.05$ for NIDDM patients; $r=0.84, P<0.05$ for nondiabetic patients) during HVC. In conclusion, (a) SGU after oral glucose administration is decreased in NIDDM as measured by both methods, and (b) SGU during the OG-CLAMP is wellcorrelated to SGU and first-pass SGU during HVC in NIDDM. The decrease in SGU in NIDDM might contribute to postprandial hyperglycemia in diabetic subjects. (J. Clin. Invest. 1997. 100:2354-2361.) Key words: OG-CLAMP • he-
\end{abstract}

Address correspondence to Jerrold M. Olefsky, M.D., Professor of Medicine, University of California San Diego and Veterans Administration Hospital, 3350 La Jolla Village Drive, San Diego, CA 92161. Phone: 619-534-6651; FAX: 619-534-6653.

Received for publication 26 September 1996 and accepted in revised form 28 August 1997.

The Journal of Clinical Investigation

Volume 100, Number 9, November 1997, 2354-2361

http://www.jci.org patic vein catheter technique - non-insulin-dependent diabetes mellitus - postprandial hyperglycemia - splanchnic glucose uptake

\section{Introduction}

Non-insulin-dependent diabetes mellitus (NIDDM) ${ }^{1}$ is characterized by insulin resistance, impaired insulin secretion, and reduced suppression of hepatic glucose production (1), factors that contribute to hyperglycemia. After oral glucose administration, the liver switches from glucose production to glucose uptake (2), and it has been shown that the splanchnic bed takes up from 9 to $60 \%$ of the orally administered glucose, depending on the method employed for measurement of splanchnic glucose uptake (SGU). It has been speculated that decreased postprandial SGU could contribute to postprandial hyperglycemia in NIDDM. Several authors using the hepatic vein catheterization (HVC) $(3,4)$ or the double-isotope technique $(5-7)$ have found increased overall systemic glucose appearance after glucose ingestion in diabetic compared with control subjects, which could result from diminished suppression of endogenous glucose output, diminished SGU, or both. However, the question of whether decreased SGU contributes to postprandial hyperglycemia in NIDDM remains unanswered.

Until recently, two methods, HVC and the double-tracer approach, have been used to study SGU in human subjects. However, both methods have several limitations regarding applicability and repetitive use in the same subjects, due mainly to the invasive nature of the HVC technique, method-related problems like glucose cycling in the double-tracer approach, and radiation exposure. We have developed recently a noninvasive technique called OG-CLAMP to measure SGU based on the combination of a hyperinsulinemic clamp and an oral glucose tolerance test during a steady state of glucose disposal. We have validated this method against the HVC technique in control and obese subjects and found an excellent correlation for SGU between both methods (8). Furthermore, we found evidence for increased SGU after oral glucose administration in nondiabetic, obese individuals, which might help prevent postprandial hyperglycemia in insulin-resistant subjects.

This study was undertaken $(a)$ to measure SGU after oral glucose administration in patients with NIDDM compared

1. Abbreviations used in this paper: BMI, body mass index; $F$, fractional extraction of glucose; $\mathrm{G}_{\mathrm{a}}$, glucose concentration in the arteries; GDR, glucose disposal rate; $\mathrm{G}_{\mathrm{hv}}$, glucose concentration in the hepatic vein; GINF, glucose infusion rate; HGO, hepatic glucose output; HVC, hepatic vein catheterization; NIDDM, non-insulin-dependent diabetes mellitus; NSGB, net splanchnic glucose balance; OGL, oral glucose load; OGR, oral glucose resorption; SBF, splanchnic blood flow; SG, total glucose uptake by splanchnic area per unit of time; $\mathrm{SGU}$, splanchnic glucose uptake; $\mathrm{T}_{\mathrm{abs}}$, time required for absorption of the glucose load. 
with age- and weight-matched subjects during HVC, and (b) to investigate the validity and applicability of the OG-CLAMP approach in diabetic subjects.

\section{Methods}

\section{Subjects}

Six nondiabetic subjects (group C: males, age $41.0 \pm 2.1 \mathrm{yr}$, body mass index $[\mathrm{BMI}] 30.8 \pm 2.0 \mathrm{~kg} / \mathrm{m}^{2}$ ) and six patients with NIDDM (group D: five males, one female, age $50.5 \pm 5.3 \mathrm{yr}$, BMI $30.8 \pm 1.1 \mathrm{~kg} / \mathrm{m}^{2}$, diabetes duration $7.7 \pm 2.7 \mathrm{yr})$ matched for age $(P=0.12$ vs. group $C)$ and for BMI ( $P=1$ vs. group $\mathrm{C})$ participated in the study. All participants were admitted $3 \mathrm{~d}$ before the respective study to the San Diego Veterans Affairs Medical Center Special Diagnostic Treatment Unit, and consumed a weight maintenance diet containing $55 \%$ carbohydrate, $30 \%$ fat, and $15 \%$ protein. None of the NIDDM subjects was on insulin treatment, and oral antihyperglycemic treatment was withdrawn 3 wk before the respective studies. No subject was taking any medication known to affect glucose metabolism. The purpose, nature, and potential risks of the study were explained in detail to all subjects before obtaining their written consent. The study protocol was reviewed and approved by the Human Subjects Committee of the University of California San Diego.

\section{Experimental protocol}

All studies were performed at 8:00 a.m. after a 10-12-h overnight fast. There was an interval of at least $1 \mathrm{wk}$ between the OG-CLAMP and the HVC study. The respective experiments were carried out in random order.

\section{OG-CLAMP method}

Glucose clamp study. The glucose clamp was performed as described previously under euglycemic conditions to measure glucose uptake quantitatively and to maintain plasma glucose and serum insulin levels at required levels $(9,10)$. Using this approach, an antecubital vein was cannulated in an antegrade manner to administer infusates. A dorsal hand vein was cannulated in a retrograde fashion and kept in a warming device $\left(72^{\circ} \mathrm{C}\right)$ to facilitate venous sampling and to provide arterialized venous blood. A loading dose of insulin was administered in a logarithmically decreasing manner over the next $10-\mathrm{min}$ period, followed immediately by a constant infusion rate of $120 \mathrm{mU} / \mathrm{m}^{2} / \mathrm{min}$, for the next $360 \mathrm{~min}$. The serum glucose level was maintained at the desired level throughout the study by monitoring the glucose level at 5 -min intervals and adjusting the infusion rate of a $20 \%$ dextrose solution using a servo-control negative feedback principle. Thus, plasma glucose and insulin levels were kept constant while the glucose infusion varied, and the rate of glucose uptake assessed by the concomitant administration of 3-[ $\left.{ }^{3} \mathrm{H}\right]$ glucose served as a direct measurement of insulin effectiveness. Potassium and phosphate were given intravenously to compensate for the intracellular movement of these ions and to maintain normal serum levels.

Measurement of hepatic glucose output (HGO). Glucose turnover was quantitated by infusion of 3-[ $\left.{ }^{3} \mathrm{H}\right]$ glucose in a continuous fashion at a rate of $0.15 \mu \mathrm{Ci} / \mathrm{min} .3-\left[{ }^{3} \mathrm{H}\right]$ glucose was infused for a 4-h infusion period before all measurements. For measurement of basal hepatic glucose production rate, samples were obtained at 10-min intervals for a subsequent $30 \mathrm{~min}$. During the clamp study, measurements were made at 20-min intervals to determine both the concentration and specific activity. Changes in glucose turnover were calculated using the variable tracer (dextrose $20 \%, 200 \mu \mathrm{Ci} /$ /iter) infusion technique (11), which allows calculation of glucose disposal rate (GDR) during non-steady state conditions.

Hepatic glucose uptake study. After $3 \mathrm{~h}$ of the insulin infusion, a 75-g oral glucose load (OGL) was administered to the subjects, and the glucose level was attempted to be maintained at the previous steady state level by adjustment of the rate of glucose infusion to compensate for gastrointestinal glucose absorption. Complete ab- sorption was indicated when the preload glucose infusion set was reached again or exceeded. The rate of hepatic glucose uptake was then calculated by subtracting the decrease in glucose infusion rate (GINF) from the known amount of glucose ingested. Blood was sampled from the dorsal hand vein before the clamp, at steady state and every 15 min after administration of the OGL, to determine insulin, glucagon, and C-peptide levels.

\section{HVC study}

Under local anesthesia with $2 \%$ lidocaine, the femoral artery was punctured with an 18-gauge needle, and a 5 French Teflon catheter was introduced. The catheter tip was positioned fluoroscopically at the level of the inferior end of the sacroiliac joint so that it remained distal to the origin of the internal iliac artery. The femoral vein was punctured similarly, and a 6.5 French polyethylene catheter was advanced via the inferior vena cava into the right-side hepatic vein under fluoroscopic control. The catheter was advanced into a wedge position and then withdrawn $1-2 \mathrm{~cm} .1 \mathrm{ml}$ of contrast medium was injected to visualize the tip of the catheter and to ensure that it was positioned in an area of adequate blood flow.

Hepatic blood flow was estimated by a primed continuous infusion of indocyanine green (12). The dye infusion was initiated via an antecubital vein $75 \mathrm{~min}$ before glucose ingestion, and was continued throughout the study. Blood was sampled from the artery and the hepatic vein simultaneously at 10 -min intervals starting $45 \mathrm{~min}$ after the beginning of green dye infusion. At time zero, the subjects ingested $300 \mathrm{ml}$ of a $75 \mathrm{-g}$ glucose solution over $5 \mathrm{~min}$. Arterial and hepatic venous blood were sampled at 15 -min intervals to determine the concentrations of glucose, C-peptide, insulin, glucagon, and indocyanine green for $4 \mathrm{~h}$ after glucose ingestion.

Hepatic plasma flow was calculated by dividing the green dye infusion rate by arteriohepatic venous dye concentration difference. Hepatic blood flow was estimated by dividing hepatic plasma flow by (1 - hematocrit).

\section{Calculations}

HVC technique. The HVC technique allows measurement of the splanchnic blood flow (SBF) and the glucose concentration in the arteries $\left(\mathrm{G}_{\mathrm{a}}\right)$ and hepatic vein $\left(\mathrm{G}_{\mathrm{hv}}\right)$. Net splanchnic glucose balance (NSGB) was calculated as $S B F \cdot\left(G_{\mathrm{hv}}-G_{\mathrm{a}}\right)$. SGU during HVC was calculated by a formula reported recently (8). In the fasting state, the splanchnic bed receives glucose via the hepatic and mesenteric arteries at the systemic glucose concentration; therefore, the glucose influx to the splanchnic area is $S B F \cdot G_{\mathrm{a}}$. After administration of an OGL, the portal glucose level rises and the total glucose influx to the splanchnic area is then $S B F \cdot G_{\mathrm{a}}+$ the rate of oral glucose resorption $(O G R)$. The glucose efflux from the splanchnic bed is $G_{\mathrm{hv}} \cdot S B F$ and must equal the sum of the total glucose influx after extraction. The relation is shown by the following equation:

$S B F \cdot G_{\mathrm{hv}}=\left(S B F \cdot G_{\mathrm{a}}+O G R\right)(1-F)$,

where $F$ is the fractional extraction of glucose. Solving the equation for $F$, one obtains:

$F=\frac{S B F \cdot G_{\mathrm{a}}+O G R-S B F \cdot G_{h v}}{S B F \cdot G_{\mathrm{a}}=O G R}$

Lacking a method to measure portal glucose levels, the only unknown in this equation is $O G R$. However, assuming complete absorption of the glucose load, $O G R$ can be substituted by $O G L$. Complete absorption of the glucose load can be assumed when the fasting HGO is reached again, and the time required for absorption of the glucose load, $T_{\text {abs }}$, can then be used for calculating the integrated hepatic fractional extraction $F$ according to the following formula:

$F=\frac{\int_{0} T_{\mathrm{abs}} S B F(\mathrm{t}) G_{\mathrm{a}}(\mathrm{t}) \mathrm{dt}+O G L-\int_{0} T_{\mathrm{abs}} S B F(\mathrm{t}) G_{\mathrm{hv}}(\mathrm{t}) \mathrm{dt}}{\int_{0} T_{\mathrm{abs}} S B F(\mathrm{t}) G_{\mathrm{a}}(\mathrm{t}) \mathrm{dt}+O G L}$ 
The numerator in Eq. 2 represents the total amount of glucose taken up by the splanchnic area per unit of time (SG). Rearranging Eq. 2, one obtains the following:

$S G=F\left(S B F \cdot G_{\mathrm{a}}+O G L\right)$.

The relative amount of glucose taken up by the splanchnic area after an OGL (SGU) can be expressed as follows:

$S G U(\%)=\frac{S G}{O G L} \cdot 100$.

The HVC technique measures the net appearance of glucose on the systemic side, and therefore, includes glucose that has already passed through the splanchnic area and has not been taken up by peripheral tissues. During the OG-CLAMP method, rise of the arterial glucose level is prevented by reducing the GINF to compensate for gastrointestinal glucose absorption. Therefore, the OG-CLAMP method measures first-pass SGU, whereas the HVC method measures integrated SGU over the period of glucose absorption. As the fractional hepatic glucose extraction does not change during peripheral hyperglycemic conditions comparable to those during the HVC experiment in our patients $(4,13)$, it is valid to calculate first-pass SGU by substituting $G_{\mathrm{a}}$ in Eq. 4 by the basal arterial glucose level $\left(G_{\text {abasa }}\right)$. The total amount of glucose taken up by the splanchnic area per unit of time after the first pass $\left(S G_{\mathrm{fp}}\right)$ becomes, then, the following:

$S G_{\mathrm{fp}}=F \cdot\left(S B F \cdot G_{\mathrm{abasa}}+O G L\right)$.

The relative amount of glucose taken up by the splanchnic area after the first pass $\left(S G U_{\mathrm{fp}}\right)$ can then be calculated according to the following formula:

$S G U_{\mathrm{fp}}(\%)=\frac{S G_{\mathrm{fp}}}{O G L} \cdot 100$.

These equations do not account for residual hepatic glucose production, which has been shown to decrease to $\sim 50 \%$ of its fasting value in nondiabetic subjects (14) and to $80 \%$ in diabetic subjects (7). Consequently, the weight of this term becomes rather small relative to the total glucose influx, and has been assumed to be zero. If a finite value is assigned to it, the values for $F$ will become higher.

OG-CLAMP technique. GINF $(\mathrm{mg} / \mathrm{kg} / \mathrm{min})$ was calculated for 20-min intervals and corrected for changes in pool fraction throughout the entire experiment. In lean nondiabetic subjects, GINF was constant between 120 and $180 \mathrm{~min}$, and the mean value for GINF between 120 and 180 min was taken as the steady state for calculation. The assumption that GDR does not change during or after oral glucose administration has been validated in our previous study describing the OG-CLAMP method in more detail (8). In this study, an additional OG-CLAMP was performed in a group of subjects using a continuous $3-\left[{ }^{3} \mathrm{H}\right]$ glucose infusion, without labeling the infusate (cold GINF method). Since the plasma glucose level (and, therefore, the glucose pool) was kept constant throughout the study, any change in GDR would have led to a change in glucose specific activity of the 3 - $\left[{ }^{3} \mathrm{H}\right]$ glucose tracer. However, no change in specific activity occurred during or after the period of glucose absorption, and, therefore, GDR remained constant after the pre-OGL steady state at $180 \mathrm{~min}$. In obese, insulin-resistant subjects, activation is slower than in lean controls (15), and, therefore, in these subjects, GINF rose slightly between 120 and $180 \mathrm{~min}$. In these subjects, the clamp was extended for an additional 40-60 min until steady state was reached before oral glucose administration. However, most diabetic subjects were substantially insulin-resistant and, therefore, did not reach steady state of glucose disposal or complete suppression of endogenous hepatic glucose production after $180 \mathrm{~min}$ of the clamp. Furthermore, GINF was not high enough to allow appropriate reduction to compensate for oral glucose appearance and still maintain euglycemia. Thus, glucose levels rose in some very resistant subjects after glucose administration despite complete discontinuation of intravenous glucose infusion. Therefore, the mean of the non-steady state GINFs before oral glucose administration and after complete oral glucose resorption, which was indicated by a sharp rise in GINF to at least pre-OGL values, was taken as insulin-mediated GINF (reference GINF) during the resorption period. To compensate for the rise in peripheral glucose levels and, thus, in the glucose pool, the reference GINF for the respective 20min intervals was corrected for these changes in pool fraction by multiplying the reference GINF by 0.061 (16).

After oral glucose administration, the respective GINFs were subtracted from the GINF of the 160-180-min interval in control subjects, and from the corrected reference GINF in diabetic patients, respectively. Multiplication of the difference in GINF with the respective time interval used for calculation $(20 \mathrm{~min})$ and the body weight yields the absolute reduction of GINF in grams of glucose. The integrated reduction of GINF over the time required for glucose absorption was then subtracted from the known amount of glucose given $(75 \mathrm{~g})$. Although the assumption that all of the $75 \mathrm{~g}$ of glucose are absorbed is substantially accurate no convenient method exists to measure this quantitatively. Therefore, it is possible that a small amount of oral glucose remains unabsorbed. In this event, it would be a small fraction of the total glucose load, and would probably be the same in both groups. If so, then this would not affect any of our interpretations or conclusions about differences in hepatic glucose uptake between normal and NIDDM patients, but would simply alter slightly our calculation of the percentage of the glucose load taken up by the splanchnic bed, since SGU was expressed as percentage of glucose retained by the splanchnic area of the administered glucose load.

\section{Measurements}

Glucose was measured with an automated glucose analyzer (Yellow Springs Instrument Co., Yellow Springs, OH). Insulin was assayed by a double-antibody RIA according to the method of Desbuquois and Aurbach (17). C-peptide (18) and glucagon (19) were measured as described previously. Indocyanine green was analyzed by spectrophotometer after precipitation with sodium desoxycholate (20).

\section{Statistical analysis}

All data are presented as mean \pm SEM. All statistical comparisons between different groups were performed by the unpaired $t$ test analysis. Changes from baseline within any given group were evaluated by the paired $t$ test. Coefficients of correlation were determined with the StatView program (Abacus Concepts, Inc., Berkeley, CA).

\section{Results}

HVC technique. Arterial glucose $(A)$, insulin $(B)$, and glucagon $(C)$ levels after the OGL are shown in Fig. 1, depicting the high glucose and low insulin levels in group D compared with group C. Basal glucagon levels were comparable between the two groups (132 $\pm 23 \mathrm{ng} /$ liter in group C, and $111 \pm 10 \mathrm{ng} / \mathrm{liter}$ in group $\mathrm{D}$, respectively, $P=0.43$ ) and tended to suppress in group C over the initial $180 \mathrm{~min}$, returning to basal by $240 \mathrm{~min}$.

After oral glucose administration, the SBF increased from $1,261 \pm 67 \mathrm{ml} / \mathrm{min}$ in group $\mathrm{C}$ and $1,301 \pm 55 \mathrm{ml} / \mathrm{min}$ in group $\mathrm{D}$ at basal, to a peak of $1,868 \pm 154 \mathrm{ml} / \mathrm{min}$ in group $\mathrm{C}$ and $1,648 \pm 193 \mathrm{ml} / \mathrm{min}$ in group $\mathrm{D}$ after $30 \mathrm{~min}$, and then declined to $1,219 \pm 61 \mathrm{ml} / \mathrm{min}$ in group $\mathrm{C}(P=0.70 \mathrm{vs}$. basal $)$ and $1,317 \pm 169 \mathrm{ml} / \mathrm{min}$ in group $\mathrm{D}(P=0.92 \mathrm{vs}$. basal $)$ at $180 \mathrm{~min}$. The time course of NSGB after oral glucose administration paralleled that of SBF, and is shown in Fig. 2. NSGB rose from basal $135.8 \pm 12.3 \mathrm{mg} / \mathrm{min}$ for group $\mathrm{C}$ and $139.8 \pm 18.9 \mathrm{mg} / \mathrm{min}$ for group D to a peak of $692.3 \pm 139.1 \mathrm{mg} / \mathrm{min}$ for group C at 30 min and $767.1 \pm 101.2 \mathrm{mg} / \mathrm{min}$ for group D at $45 \mathrm{~min}$, and subsequently returned to values that were not significantly different from basal values $(210 \pm 94 \mathrm{mg} / \mathrm{min}$ for group $\mathrm{C}, P=0.46$ 

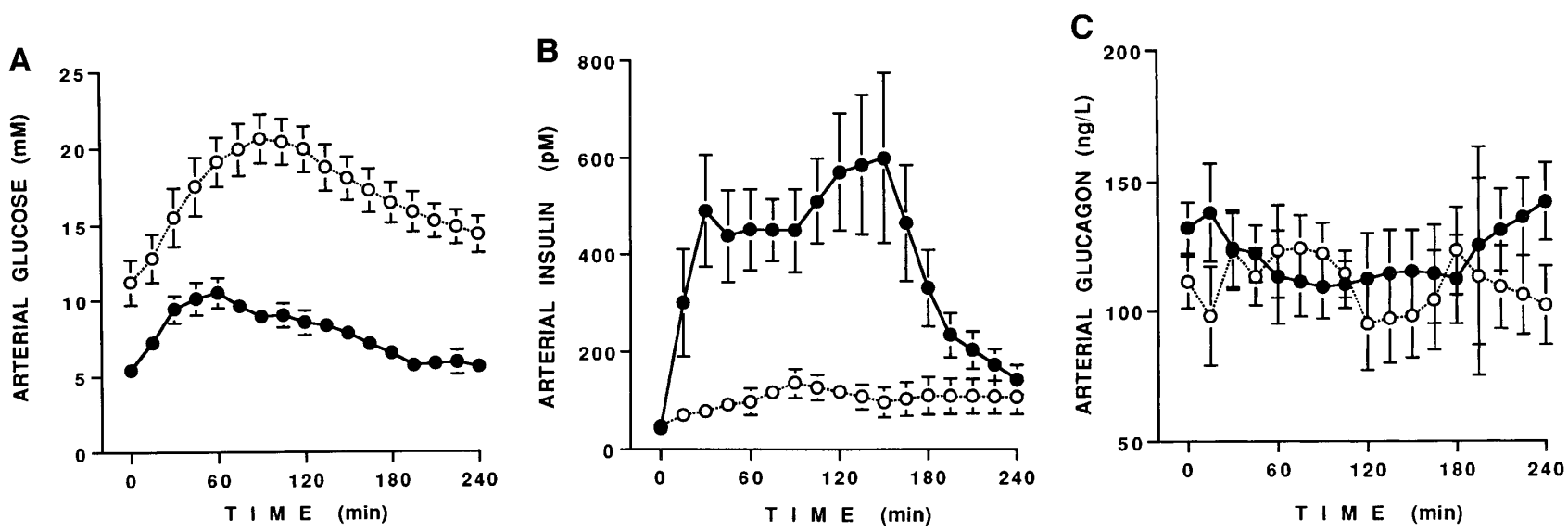

Figure 1. Arterial glucose $(A)$, insulin $(B)$, and glucagon $(C)$ levels after oral glucose administration during HVC. $\bullet$, Controls. $\bigcirc$, Diabetic patients.

vs. basal, and $109 \pm 30 \mathrm{mg} / \mathrm{min}$ for group $\mathrm{D}, P=0.55 \mathrm{vs}$. basal, at $180 \mathrm{~min})$. Complete glucose absorption was indicated when SBF returned to preload values and NSGB was not significantly different from the basal value. The mean time required for glucose absorption was $165 \pm 11 \mathrm{~min}$ for group $\mathrm{C}$ and $143 \pm 10$ min for group $\mathrm{D}(P=0.16$, group $\mathrm{C}$ vs. group $\mathrm{D})$.

Cumulative NSGB output was $46.7 \pm 4.4 \mathrm{~g}$ for group $\mathrm{C}$ and $73.1 \pm 5.1 \mathrm{~g}$ for group D $(P<0.01)$, SGU was $37.8 \pm 5.9 \%$ for group C and $7.4 \pm 2.1 \%$ for group D of the 75 -g glucose load $(P<$ 0.001 , Fig. 3), and first-pass SGU was $26.5 \pm 5.1 \%$ for group $\mathrm{C}$ and $4.7 \pm 1.7 \%$ for group D $(P<0.01)$.

OG-CLAMP technique. Mean isotopically determined steady state GDR during the last hour of the glucose clamp before oral glucose administration was $7.19 \pm 0.77 \mathrm{mg} / \mathrm{kg} / \mathrm{min}$ for group $\mathrm{C}$ and $4.95 \pm 0.94 \mathrm{mg} / \mathrm{kg} / \mathrm{min}$ for group D. Endogenous $\mathrm{HGO}$ was $2.22 \pm 0.26 \mathrm{mg} / \mathrm{kg} / \mathrm{min}$ for group $\mathrm{C}$ and $2.74 \pm 0.18$ $\mathrm{mg} / \mathrm{kg} / \mathrm{min}$ for group $\mathrm{D}$ in the basal state $(P=0.06)$, and was suppressed by $91 \pm 4 \%$ to a steady state level of $0.23 \pm 0.11 \mathrm{mg} /$ $\mathrm{kg} / \mathrm{min}$ in group C and by $64 \pm 6 \%$ to $0.98 \pm 0.17 \mathrm{mg} / \mathrm{kg} / \mathrm{min}$ in group D after $180 \mathrm{~min}$, as measured with the variable tracer in-

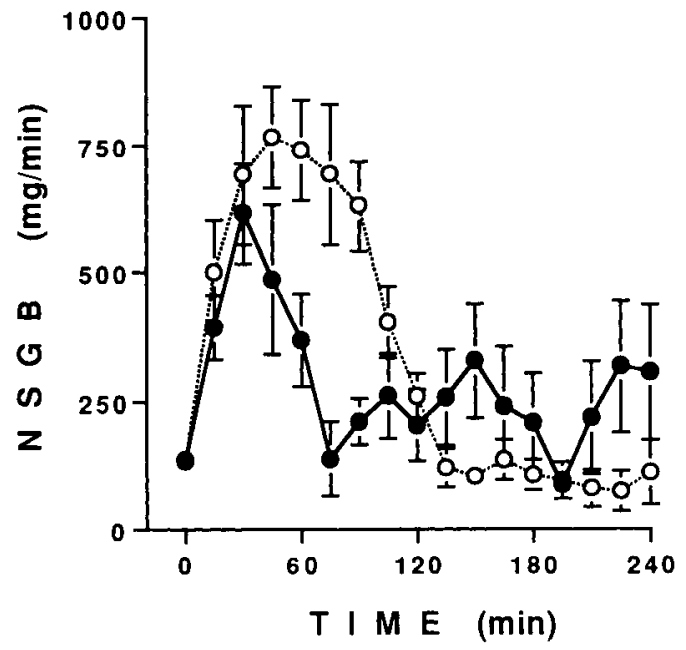

Figure 2. NSGB after oral glucose administration during HVC. Controls. $\bigcirc$, Diabetic patients. fusion method. The time course of the GINF is shown in Fig. 4. Complete glucose absorption was indicated by the return of the actual GINF to the value before the OGL. The time required for GINF to return to the pre-OGL value was $167 \pm 11$ min in group C and $149 \pm 10 \mathrm{~min}$ in group D. The glucose specific activity is used only for calculation of the suppression of HGO before OGL. After the appearance of unlabeled orally administered glucose into the circulation, the specific activity is altered rapidly and cannot be used for calculations. Estimation of SGU during the OG-CLAMP method does not require tracer methods, a potential advantage to this approach.

Fig. $5 A$ shows glucose levels during the OG-CLAMP. In group $\mathrm{C}$, plasma glucose after the OGL remained constant (5.3 $\pm 0.1 \mathrm{mM}$ before OGL, 5.6 $\pm 0.2 \mathrm{mM}$ at $240 \mathrm{~min}$, and $5.3 \pm 0.4 \mathrm{mM}$ at $360 \mathrm{~min}$ ). In group D, plasma glucose increased after the OGL from $5.1 \pm 0.2 \mathrm{mM}$ at steady state to $9.0 \pm 1.5$ $\mathrm{mM}$ at $255 \mathrm{~min}$, and declined subsequently to $5.3 \pm 0.1 \mathrm{mM}$ at $360 \mathrm{~min}$. C-peptide levels (Fig. 5 B) decreased after the initiation of the clamp from $752 \pm 136 \mathrm{ng} /$ liter in group $\mathrm{C}$ and $546 \pm 95 \mathrm{ng} / \mathrm{liter}$ in group $\mathrm{D}$, at basal, to $376 \pm 57 \mathrm{ng} / \mathrm{liter}$ in group C and $149 \pm 38 \mathrm{ng} /$ liter for group D at $180 \mathrm{~min}$, reflecting suppression of endogenous insulin secretion due to the exoge-

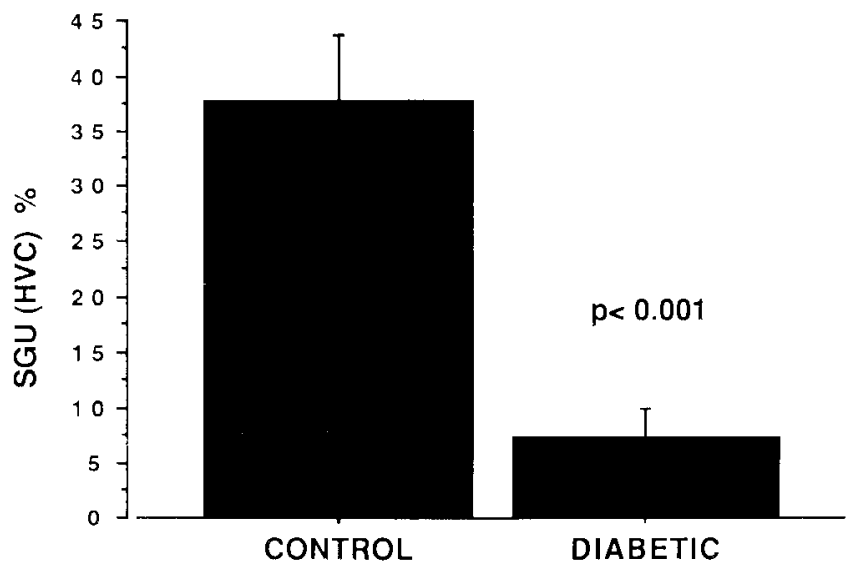

Figure 3. SGU during HVC in control and diabetic subjects. 


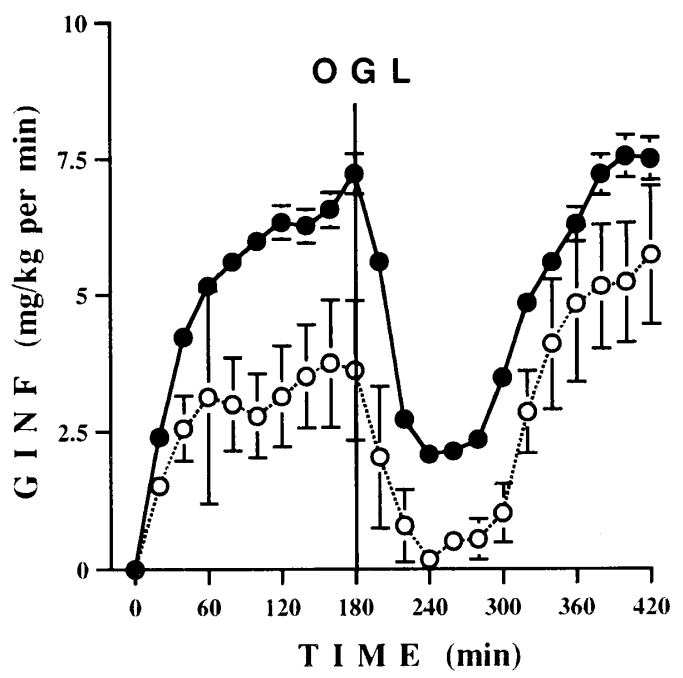

Figure 4. GINF during OG-CLAMP. @, Controls. $\bigcirc$, Diabetic patients.

nous insulin infusion. Insulin secretion was stimulated significantly during oral glucose absorption in both groups after the OGL, returning to steady state levels after $180 \mathrm{~min}$. Serum glucagon levels (Fig. 5 C) decreased from basal $150 \pm 27 \mathrm{ng} /$ liter in group C and $121 \pm 13 \mathrm{ng} /$ liter in group $\mathrm{D}$, to $114 \pm 12 \mathrm{ng} /$ liter in group $\mathrm{C}(P<0.01 \mathrm{vs.} \mathrm{basal})$ and $84 \pm 16 \mathrm{ng} /$ liter in group $\mathrm{D}(P<$ 0.01 vs. basal) at steady state, and did not change significantly after oral glucose administration.

Cumulative HGO after the OGL was $57.5 \pm 1.9 \mathrm{~g}$ for group $\mathrm{C}$ and $76.5 \pm 5.5 \mathrm{~g}$ for group $\mathrm{D}(P<0.01)$, and SGU was $23.4 \pm 2.5 \%$ for group $\mathrm{C}$ and $3.9 \pm 1.7 \%$ for group $\mathrm{D}(P<$ 0.0001, Fig. 6).

Correlations between HVC and OG-CLAMP for groups $C$ and $D$. Highly significant correlations were found between the OG-CLAMP versus the HVC technique, indicating that both methods provided comparable information. Thus, the correlations between splanchnic glucose output measured by HVC versus the OG-CLAMP method were $0.94, P<0.001$ in controls, and $0.98, P<0.001$ in NIDDM patients. For SGU, the values were $r=0.97, P<0.001(r=0.94, P<0.01$ for group $\mathrm{C}$,

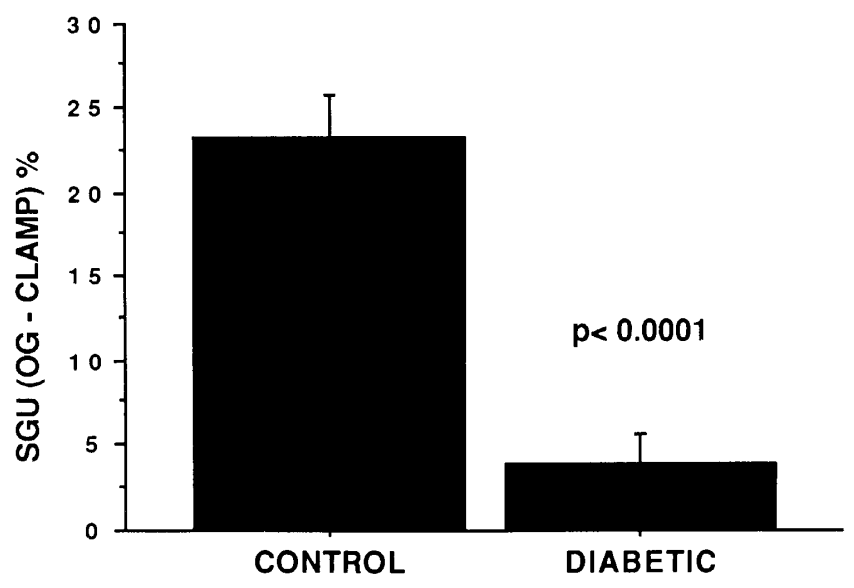

Figure 6. SGU during OG-CLAMP in control and diabetic subjects.

and $r=0.87, P<0.05$ for group D, Fig. $7 A$ ), and for first-pass SGU, values were $r=0.93, P<0.05(r=0.84, P<0.05$ for group $\mathrm{C}$, and $r=0.87, P<0.05$ for group $\mathrm{D}$, Fig. $7 B$ ).

\section{Discussion}

It is widely recognized that abnormalities of hepatic glucose metabolism play an important role in the pathophysiology of NIDDM (3-6). However, most attention is focused on the increased rates of basal hepatic glucose production, which have been widely described (1), and represent a major cause of fasting hyperglycemia. However, in addition to being the major source of glucose production during the interprandial state, after glucose ingestion, the liver is also an important site of glucose disposition $(2-4,6,7,14)$. Thus, abnormalities of hepatic glucose uptake could also play a role in the pathophysiology of NIDDM. In this study, we have used two methods to measure hepatic glucose uptake of orally ingested glucose in normal and NIDDM patients. The first method is the standard HVC approach, which allows measurement of glucose balance over the splanchnic bed (3). The second method uses an approach we have validated recently and called the OG-CLAMP technique (8). The latter is a relatively noninvasive procedure in
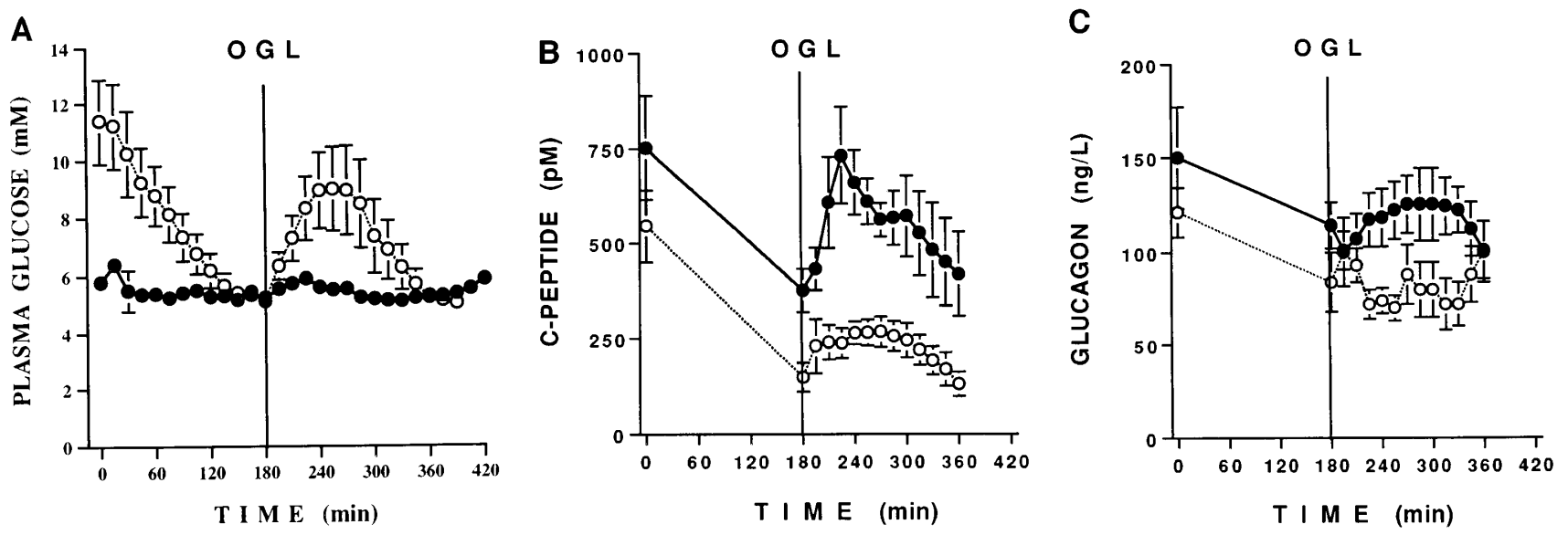

Figure 5. Plasma glucose $(A)$, serum C-peptide $(B)$, and glucagon $(C)$ levels during OG-CLAMP. $\bullet$, Controls. $\bigcirc$, Diabetic patients. 

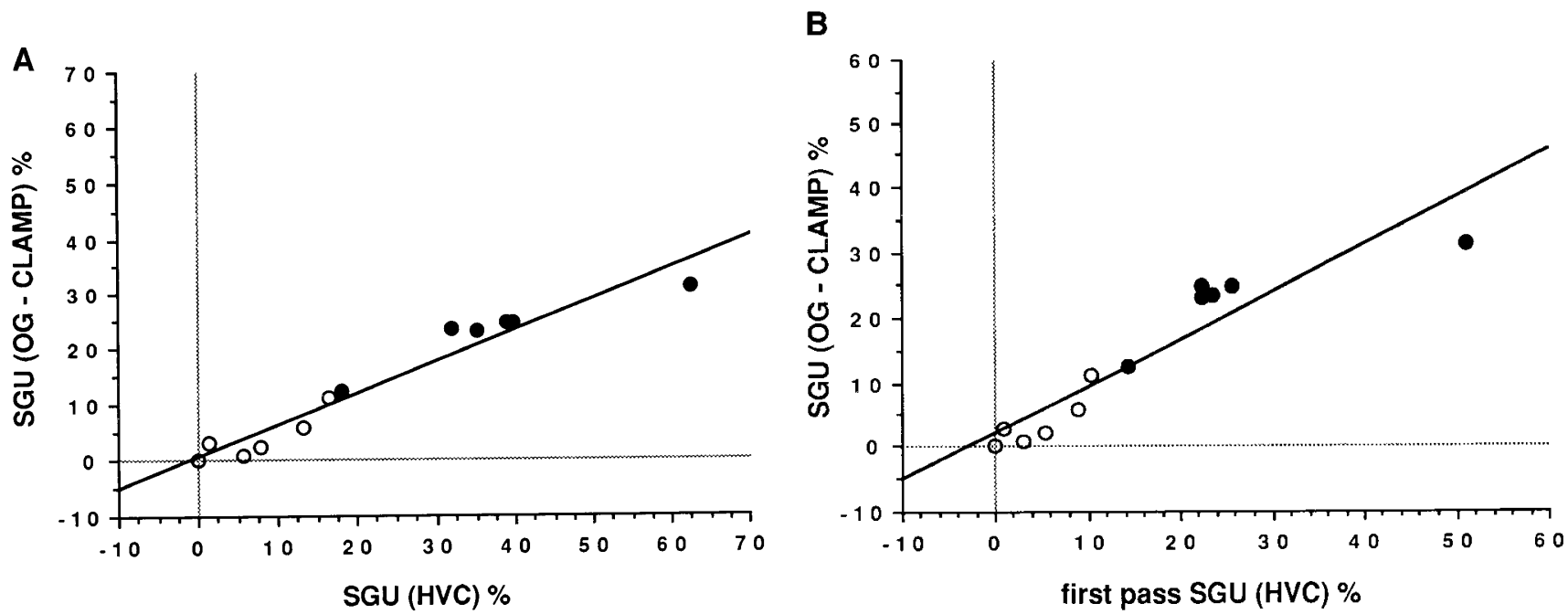

Figure 7. (A) Correlation between SGU during OG-CLAMP and SGU during HVC. (B) Correlation between SGU during OG-CLAMP and first-pass SGU during HVC. $\bullet$, Controls. $\bigcirc$, Diabetic patients.

which an OGL is administered during the steady state of a hyperinsulinemic euglycemic clamp, and a decrease in exogenous glucose infusion during the period of oral glucose absorption is taken as a measure of oral glucose that bypasses the liver and is delivered to the systemic circulation. Both of these techniques have given quite comparable results, showing a very marked decrease in hepatic glucose uptake in NIDDM subjects. This reduction in hepatic glucose uptake results in a greater proportion of orally ingested glucose during the systemic circulation, and can lead directly to exacerbation of postprandial hyperglycemia, which is a characteristic of NIDDM subjects.

In previous reports, SGU in NIDDM patients has been measured by HVC or by a double-tracer approach. Using HVC, Felig et al. (3) found diminished SGU in NIDDM patients, as did Ferrannini et al. (14). Using a dual isotope technique, Firth et al. found increased appearance of ingested glucose in NIDDM, suggesting an important role for decreased SGU in postprandial hyperglycemia (6). However, in a more recent study, the same group found that SGU was increased in NIDDM patients compared with controls (5). Similarly, Mitrakou et al. found that SGU was higher in NIDDM subjects (7). From these double-tracer studies, it has been concluded that the decrease in SGU in NIDDM is small, and that impaired suppression of endogenous hepatic glucose production is the main cause of the increased systemic glucose appearance in the postprandial state in NIDDM.

Several factors may influence the calculation of SGU during HVC, such as the estimated time period over which glucose absorption occurs and potential differential suppression of ongoing endogenous hepatic glucose production. Isotopic studies have indicated that the time period required for complete absorption of an OGL ranges from 120 (21) to $240 \mathrm{~min}$ (22). To assess accurately the time period for complete glucose absorption during our HVC experiments, we have marked the end of the glucose absorption period as the time when NSGB returns to preload measurements, which indicates that glucose absorption is complete or that a small unmeasurable rate con- tinues. The possibility can be raised that the return of NSGB to baseline might represent partial suppression of endogenous HGO plus an ongoing equal degree of glucose absorption. However, such glucose fluxes would be quantitatively minor and, thus, not affect our findings of decreased SGU in diabetic patients. Additionally, the fact that the calculated SGU for nondiabetic subjects in both methods agrees with the findings of other studies using the double-tracer technology (23), and the close correlation between SGU as measured by the HVC and OG-CLAMP methods, argue strongly for the validity of our approach. Furthermore, to ensure that there was no late appearance of orally administered glucose, the HVC study was carried out for a total of $240 \mathrm{~min}$, and, as seen in Fig. 2, NSGB did not change after the assumed time required for glucose absorption of $143 \mathrm{~min}$ in diabetic patients. Thus, extension of the period for calculation of SGU would not have changed the results. Using these criteria, the calculated period for glucose absorption (165 $\pm 11 \mathrm{~min}$ for controls and 143 $\pm 10 \mathrm{~min}$ for NIDDM patients) was within the ranges reported in the literature (2123). The accuracy of this calculation was further confirmed by the measured values taken during the OG-CLAMP experiments in the same patients $(167 \pm 11 \mathrm{~min}$ in controls and $149 \pm 10 \mathrm{~min}$ in NIDDM patients). Regarding the variable of ongoing, endogenous hepatic glucose production, we repeated our calculations assuming that endogenous $\mathrm{HGO}$ was suppressed by $50 \%$ in control subjects and by $20 \%$ in the NIDDM patients (values consistent with those published in the literature [7, 22]), and even though this approach would diminish any differences in SGU between control (53.5 $\pm 7.1 \%)$ and diabetic patients $(24.7 \pm$ $6.1 \%, P<0.05$, group $\mathrm{C}$ vs. group $\mathrm{D})$, we still observed a marked reduction in SGU in NIDDM patients. Consequently, we believe that our conclusion that SGU is decreased markedly in NIDDM is valid, regardless of underlying assumptions about the time period of glucose absorption or the rate of endogenous glucose production.

In this study, we have further confirmed the finding of decreased hepatic oral glucose disposition in NIDDM by the OGCLAMP technique. This method allows the noninvasive mea- 
surement of total body glucose disposal and SGU, as well as of the time required for full absorption of an OGL in a single experiment. This technique has been validated previously against the HVC approach (8). With the OG-CLAMP method, an OGL is administered after $3 \mathrm{~h}$ of a hyperinsulinemic euglycemic clamp. The hyperinsulinemia leads to stimulated whole body GDRs as well as complete suppression of hepatic glucose production. Thus, to maintain euglycemia during the period of oral glucose ingestion, the exogenous GINF is reduced to accommodate the gastrointestinal glucose absorption. Thus, the decrease in exogenous glucose infusion is a measure of the amount of orally absorbed glucose that bypasses the liver. Consequently, by subtracting the decrease in GINF (in grams of glucose) over the period of glucose absorption from the total amount of glucose administered orally ( $75 \mathrm{~g}$ ), one can calculate the amount of the orally administered glucose that is taken up directly by the liver.

While in nondiabetic subjects, calculation of SGU is quite straightforward, this calculation is somewhat more complicated in NIDDM patients. Patients with NIDDM are insulinresistant compared with control subjects, and this leads to lower steady state GINFs during the hyperinsulinemic clamp period, and in very insulin-resistant subjects, one might not achieve full steady state by $180 \mathrm{~min}$. Although we use a rather high insulin infusion rate $\left(120 \mathrm{mU} / \mathrm{m}^{2} / \mathrm{min}\right)$ to overcome these problems, in many insulin-resistant diabetic patients, the steady state rate of exogenous glucose infusion was still relatively low. As a result, even a complete reduction in exogenous glucose infusion does not accommodate fully the incoming glucose load, and plasma glucose levels rise. This increase in the plasma glucose pool leads to a mass action increase in glucose disposal. Under these circumstances, posthepatic glucose delivery of the OGL is equal to the reduction in the exogenous glucose infusion plus the mass action-induced increase in glucose disposal subtracted from the 75-g OGL. Fortunately, the rises in plasma glucose level during the OG-CLAMP were modest and within the range at which glucose clearance is proportional to glucose concentration. As stated in Methods, in each individual NIDDM patient whose plasma glucose levels increased during the OG-CLAMP, SGU was calculated using this method. While this might introduce some error, the excellent correlation for SGU between the OG-CLAMP and the HVC technique is in favor of the validity of our assumptions, and indicates that if there is any error introduced by this calculation, it is likely to be small and not systematic. This method also assumes that endogenous hepatic glucose production is suppressed completely by the maintenance of hyperinsulinemia. Although this is likely to be correct, even if some degree of residual hepatic glucose production existed in the insulinresistant NIDDM patients, the absolute magnitude of glucose production would be trivial compared with the overall rate of glucose flux during the OG-CLAMP. Furthermore, to the extent that endogenous HGO would be higher in NIDDM patients versus controls during the OG-CLAMP, this would lead to even lower SGU, and would tend to widen the differences in SGU between control and diabetic patients. Thus, the differences in SGU between the groups would be even greater, to the extent that endogenous HGO exists in the NIDDM patients.

The two approaches we have used to measure SGU have yielded remarkably comparable results. Thus, we have found excellent correlation between the SGU values calculated dur- ing HVC and those calculated during the OG-CLAMP. The absolute values of SGU are somewhat higher during HVC than OG-CLAMP because the HVC technique measures the net appearance of glucose on the systemic side, which includes glucose that has already passed through the splanchnic area, has not been taken up by the peripheral tissues, and returns for subsequent passes through the splanchnic bed. Thus, the OGCLAMP method measures first-pass SGU, whereas the HVC method measures integrated SGU over the entire period of glucose absorption. Interestingly, when first-pass SGU was calculated from the HVC experiments, the values were nearly identical to those obtained during the OG-CLAMP, and the values for SGU from either method were very well correlated (Fig. 7) in both the control and NIDDM patients.

Although our studies show clearly that SGU is decreased markedly in NIDDM patients, the mechanisms underlying this abnormality have yet to be defined. Along these lines, Magnusson et al. have used recently an MRI approach to demonstrate a decrease in glycogen concentration in the livers of NIDDM patients (24). This could be associated with an increase in intracellular free glucose concentrations or an increase in glucose-6-phosphate, both of which effects would cause a decrease in net hepatic glucose uptake. According to this line of reasoning, a decreased hepatic uptake, which we have demonstrated in these studies, would be a reflection of increased intrahepatic gluconeogenesis and/or glycogenolysis, both of which result in an increase of glucose-6-phosphate levels. Although other mechanisms are possible, the above scenario would suggest that decreased SGU in NIDDM is secondary to previously recognized derangements in intrahepatic glucose metabolism. Although this abnormality might be secondary, this does not diminish the importance of decreased SGU as a contributor to the pathophysiology of NIDDM. Obviously, a decrease in SGU of orally ingested carbohydrate would contribute to postprandial hyperglycemia. Based on our findings, the defect in SGU in NIDDM could lead to a $25-30 \%$ increase in the amount of glucose delivered to the systemic circulation. Since these patients are insulin-resistant and have decreased insulin secretion, this increased delivery of glucose to peripheral tissues would have a significant impact on the exacerbation of postprandial hyperglycemia. The patients included in this study had substantial fasting hyperglycemia and decreased insulin secretion. Thus, at this point, we cannot generalize our findings to NIDDM patients with mild degrees of hyperglycemia and relative postprandial hyperinsulinemia.

\section{Acknowledgments}

This work was supported by National Institutes of Health grant DK33649, National Institutes of Health General Clinical Research Center grant RR-00827, and by the Medical Service, Department of Veteran Affairs, Veterans Administration Medical Center, San Diego, CA. B. Ludvik was the recipient of a Max-Kade Foundation Postdoctoral Fellowship Award, and was on leave from the University of Vienna, Austria.

\section{References}

1. DeFronzo, R.A. 1988. Lilly lecture 1987. The triumvirate: beta-cell, muscle, and liver. A collusion responsible for NIDDM. Diabetes. 37:667-687.

2. Felig, P., J. Wahren, and R. Hendler. 1975. Influence of oral glucose ingestion on splanchnic glucose and gluconeogenic substrate metabolism in man. Diabetes. 24:468-475.

3. Felig, P., J. Wahren, and R. Hendler. 1978. Influence of maturity-onset 
diabetes on splanchnic glucose balance after oral glucose ingestion. Diabetes. 27:121-126.

4. Ferrannini, E., J. Wahren, P. Felig, and R.A. DeFronzo. 1980. The role of fractional glucose extraction in the regulation of splanchnic glucose metabolism in normal and diabetic man. Metabolism. 29:28-35.

5. Butler, P.C., and R.A. Rizza. 1991. Contribution to postprandial hyperglycemia and effect on initial splanchnic glucose clearance of hepatic glucose cycling in glucose-intolerant or NIDDM patients. Diabetes. 40:73-81.

6. Firth, R., P. Marsh, I. Hansen, and R. Rizza. 1986. Postprandial hyperglycemia in patients with non-insulin-dependent diabetes mellitus: roles of hepatic and extrahepatic tissues. J. Clin. Invest. 77:1525-1532.

7. Mitrakou, A., D. Kelley, T. Veneman, T. Jenssen, T. Pangburn, J. Reilly, and J. Gerich. 1990. Contribution of abnormal muscle and liver glucose metabolism to postprandial hyperglycemia in NIDDM. Diabetes. 39:1381-1391.

8. Ludvik, B., J.J. Nolan, A. Roberts, J. Baloga, M. Joyce, J.M. Bell, and J.M. Olefsky. 1995. A noninvasive method to measure splanchnic glucose uptake after oral glucose administration. J. Clin. Invest. 95:2232-2238.

9. Olefsky, J. 1981. Insulin resistance and insulin action: an in vitro and in vivo perspective. Diabetes. 30:148-162.

10. Olefsky, J.M., and O.G. Kolterman. 1981. Mechanisms of insulin resistance in obesity and non-insulin-dependent (type II) diabetes. Am. J. Med. 70: $151-168$

11. Molina, J.M., A.D. Baron, S.V. Edelman, G. Brechtel, P. Wallace, and J. Olefsky. 1990. Use of a variable tracer infusion method to determine glucose turnover in man. Am. J. Physiol. 258:E16-E23.

12. Rowell, L.B., J.R. Blackmon, and R.A. Bruce. 1964. Indocyanine green clearance and estimated hepatic blood flow during mild to maximal exercise in upright man. J. Clin. Invest. 43:1677-1690.

13. DeFronzo, R.A., E. Ferrannini, R. Hendler, P. Felig, and J. Wahren. 1983. Regulation of splanchnic and peripheral glucose uptake by insulin and hyperglycemia in man. Diabetes. 32:35-45.
14. Ferrannini, E., D.C. Simonson, L.D. Katz, G. Reichard, S. Bevilacqua, E.J. Barrett, M. Olsson, and R.A. DeFronzo. 1988. The disposal of an oral glucose load in patients with non-insulin-dependent diabetes. Metabolism. 37:79-85.

15. Prager, R., P. Wallace, and J.M. Olefsky. 1986. In vivo kinetics of insulin action on peripheral glucose disposal and hepatic glucose output in normal and obese subjects. J. Clin. Invest. 78:472-481.

16. Bergmann, R.N., D.T. Finegood, and M. Ader. 1985. Assessment of insulin sensitivity in vivo. Endocr. Rev. 6:45-86.

17. Desbuquois, B., and G.D. Aurbach. 1971. Use of polyethylene glycol to separate free and antibody-bound peptide hormones in radioimmunoassays. $J$. Clin. Endocrinol. Metab. 33:732-738.

18. Faber, O.K., C. Binder, J. Markussen, L.G. Heding, V.K. Naithani, H Kuzuya, B.P. Blix, D.L. Horwitz, and A.H. Rubenstein. 1978. Characterization of seven C-peptide antisera. Diabetes. 27:170-177.

19. Unger, R., A. Eisenhart, M. McCall, and L. Madison. 1961. Glucagon antibodies and an immunoassay for glucagon. J. Clin. Invest. 48:1280-1289.

20. Gasic, S., C.H. Kleinbloesem, G. Heinz, and W. Waldhäusl. 1991. Contribution of splanchnic and peripheral vascular tissues to the disposal of angiotensin-II and to regional conversion rates of angiotensin-I: a pilot study in humans. J. Cardiovasc. Pharmacol. 17:615-620.

21. Cummins, A.J. 1952. Absorption of glucose and methionine from the human intestine. Influence of the glucose concentration in the blood and the intestinal lumen. J. Clin. Invest. 31:928-937.

22. Radziuk, J., T.J. McDonald, D. Rubenstein, and J. Dupre. 1978. Initial splanchnic extraction of ingested glucose in normal man. Metabolism. 27:657-669.

23. Consoli, A. 1992. Role of liver in pathophysiology of NIDDM. Diabetes Care. 15:430-441.

24. Magnusson, I., D.L. Rothman, L. Katz, R.G. Shulman, and G.I. Shulman. 1992. Increased rate of gluconeogenesis in type II diabetes mellitus. $J$. Clin. Invest. 90:1323-1327. 